\title{
Germanica
}

\section{Villy Sørensen: Initiator des dänischen Prosamodernismus}

Villy Sørensen, initiateur de la prose moderniste au Danemark

\section{Hedwig Reuter}

\section{OpenEdition}

1 Journals

Édition électronique

URL : http://journals.openedition.org/germanica/1492

DOI : $10.4000 /$ germanica. 1492

ISSN : 2107-0784

Éditeur

Université de Lille

\section{Édition imprimée}

Date de publication : 1 juin 1993

Pagination : 91-109

ISSN : 0984-2632

\section{Référence électronique}

Hedwig Reuter, «Villy Sørensen: Initiator des dänischen Prosamodernismus », Germanica [Online], 12 | 1993, Online erschienen am: 06 Juli 2012, abgerufen am 06 Oktober 2020. URL : http:// journals.openedition.org/germanica/1492; DOI : https://doi.org/10.4000/germanica.1492

Ce document a été généré automatiquement le 6 octobre 2020.

(c) Tous droits réservés 


\title{
Villy Sørensen: Initiator des dänischen Prosamodernismus
}

Villy Sørensen, initiateur de la prose moderniste au Danemark

\author{
Hedwig Reuter
}

\section{Modernismus : Definition}

1 Die dänische Literaturforscherin Pil Dahlerup gibt in dem Artikel «Hvad er modernisme?» (in Modernismen i skandinavisk litteratur, Trondheim, 1991) einige präzise Definitionen, die dem Modernismus als sowohl epochalem, wie kunsttheoretischem und haltungsmäßigem Phänomen Rechnung tragen. Diese Definitionen scheinen uns für die Darstellung der vielseitigen Rolle Villy Sørensens im dänischen Modernismus von großem Nutzen. Dahlerup definiert den Modernismus als die spezifisch künstlerische Ausformung der «modernen» Haltung. Diese Kunst entsteht zuerst in Frankreich in der Mitte des 19. Jahrhunderts. In Europa erreicht sie ihren Höhepunkt um 1910-1930, und erst in den 1950 er-1960 er Jahren erfolgt ihr verspäteter Durchbruch in Dänemark.

Die Begriffe «modern » und « Modernität » versteht Dahlerup als modale Begriffe, die weder an eine bestimmte Zeit noch an eine bestimmte künstlerische Ausformung gebunden sind, sondern eine Haltung bezeichnen, die « das Neue repräsentiert, die dieses Neue als unumgänglich erachtet und es in ein Gegensatzverhältnis zu einer oder mehreren früheren Positionen setzt ». Diese Haltung ist Dahlerup zufolge das Gegenteil der historischen Haltung, in der das Neue gerade aus dem Vergangenen seine Berechtigung erhält. "Modernes» und «historisches» Bewußtsein sind deshalb miteinander unvereinbar, obwohl sie einander bedingen, denn « indem der "moderne" Mensch die Vergangenheit abtrennt, trennt er sich selber vom Jetzt und von der Zukunft $a b$ ». Die unlösbaren Konflikte, die die Stellung des «modernen» Menschen zwischen vor und jetzt mit sich bringt, sind daher das Hauptcharakteristikum der Modernität. 
3 Neben dem modalen Begriff «Modernität» und dem kunsttheoretischen Begriff «Modernismus» benutzt Dahlerup in ihrem Artikel ebenfalls die epochale Bezeichnung "Der moderne Durchbruch» («det moderne gennembrud»). Unter «Modernem Durchbruch» versteht sie «ein historisch konkretes Beispiel von "Modernität" ", das in Skandinavien um 1870 in Erscheinung tritt. Dahlerup benutzt den Begriff aber nicht ausschließlich zur Bezeichnung der Zeit von 1870 bis 1890, die durch das Wirken Georg Brandes' charakterisiert ist, sondern sie umfaßt damit die gesamte Epoche bis 1980.

4 Der «Moderne Durchbruch» charakterisiert sich Dahlerup zufolge durch einen Durchbruch (und vorausgehenden Zusammenbruch) auf politischer, produktionsmäßiger, psychologischer, existentieller... Ebene. Der zentrale Aspekt dieses Durchbruchs für die literaturtheoretische und -historische Betrachtung ist der existentielle Aspekt : die Säkularisierung der Welt. Dahlerup sieht hier den wirklichen Anfang einer neuen Epoche, denn zum ersten Mal wird dem Menschen klar, daß er selber und die Welt nicht geschaffen sind, sondern sich zu dem, was sie sind, entwickelt haben. Die bekannte Metapher für diese radikale Umwälzung des menschlichen Selbstund Weltverständisses ist der « Tod Gottes ». Der Kunst wurde durch diese Umwälzung der Boden weggezogen, auf dem sie bisher operiert hatte : sie konnte nicht länger Abbild einer transzendentalen Wirklichkeit sein, sei diese nun metaphysischchristlicher Art (wie in der Kunst des Mittelalters) oder eine sonstige höhere Wirklichkeit, die sich ästhetisch bereits vorwegnehmen ließe (wie z.B. in der deutschen Klassik). Die Künstler der modernen Epoche zogen Dahlerup zufolge aus dem Verschwinden der Transzendenz ihre Konsequenzen, aber in zwei völlig verschiedene Richtungen. Die einen verlegten sich ganz auf die diesseitige Wirklichkeit, die sie als einzige Existenzmöglichkeit anerkannten: literarisch gab sich diese Haltung im Realismus und in dessen Ausläufern, dem Naturalismus und Impressionismus Ausschlag (den Symbolismus der modernen Epoche nennt Dahlerup einen «Rückfall » in den Transzendentismus). Für die andern verlor nicht nur die Transzendenz ihre Gültigkeit für die menschliche Existenz, sondern auch die immanente Wirklichkeit: diese Künstler sind Dahlerup zufolge die Modernisten. Der literarische Modernismus ist somit die Literatur der modernen Epoche, deren existenzieller Ausgangspunkt der «Tod Gottes » und der « Tod der Wirklichkeit » ist, was auf der Ebene des literarischen Ausdrucks dem «Tod des Symbols » und dem «Tod der Mimesis » entspricht. Deshalb definiert Dahlerup die Modernisten als die «Gruppe von Dichtern, die nichts haben, worüber sie schreiben können, und die keine Art und Weise haben, auf die sie schreiben können, und die trotzdem schreiben». (Dahlerup, in Modernismen $i$ skandinavisk litteratur, S. 29). Ihr Ausgangspunkt ist die Erfahrung der absoluten Sinnlosigkeit der Welt.

\section{Villy Sørensen als Frontfigur des dänischen Modernismus}

5 Villy Sørensen ist der Denker und Schriftsteller, dessen Werk über die drei Bereiche des oben umrissenen « modernen » Phänomens deckt, und der in entscheidender Weise zu dessen Verständnis in Dänemark beigetragen hat. Durch die Erzählungen «Sære historier " (1953) leitet er in seinem Land die Wende vom realistischen Roman zur modernistischen Prosa ein. Es folgen zwei weitere Bände Erzählungen: «Ufarlige 
historier », 1955 (Ungefährliche Geschichten) und « Formynder fortællinger », 1963 ("Vormundschaftserzählungen », 1968).

6 In den gleichen Jahren, untersucht er in seinen Essays das, was wir oben mit Pil Dahlerups Begriff «Modernität» bezeichnet haben: die Empfindung eines unüberbrückbaren Konflikts zwischen alt und neu, vor und jetzt. Dieser Konflikt, der in den Erzählungen bildhaften Ausdruck fand, steht auch am Anfang von Sørensens theoretischem Denken, dessen Grundbegriffe und -themen er in "Digtere og dæmoner » (1959) zum ersten Mal formuliert. Sørensen entwickelt seine Philosophie in einem kritischen Dialog mit der modernen europäischen Philosophie seit der Mitte des 19. Jahrhunderts. Die Originalität seines eigenen Denkens liegt in der Herausstellung der Begriffe "Deutung" (symbolische Auslegung des Lebens) und "Bewertung" (intellektuelle, theoretische Stellungnahme) als grundlegenden Erkenntnisstrukturen. Bei der Entwicklung seiner Theorie nimmt Sørensen Stellung zu Figuren wie Kierkegaard, Nietzsche, Marx, Freud, Jung, usw. ; dadurch trägt er zur Aktualisierung und Neueinschätzung eines umfangreichen modernen Gedankenguts in Dänemark bei. Von andern Denkern nehmen die Dänen durch Villy Sørensens Besprechungen überhaupt erst Kenntnis : z.B. Heidegger, dessen Philosophie Sørensen so darstellt, daß - um eine Formulierung Niels Barfoeds $\mathrm{zu}$ benutzten - «auch andere als Berufsphilosophen ihn verstehen können " (Niels Barfoed, "Danske digtere i det 20. Århundrede ", Band 3, 1966, S. 466), und Hermann Broch, über den Sørensen bereits $1956^{1}$ einen Aufsatz veröffentlicht. Die Affinität Sørensen für Broch ist u.a. dadurch bedingt, daß beide sowohl Dichter und Philosoph sind und durch diese Doppelexistenz die Erkenntnismöglichkeiten beider Disziplinen gegeneinander abwägen können. Dabei erhält sowohl bei Broch wie bei Sørensen die Kunst den Vorrang vor der Philosophie.

Sørensens Hauptwerk "Digtere og dæmoner" ist denn auch an erster Stelle ein literaturtheoretisches und -kritisches Werk (für das ihm übrigens der dänische « Kritikerpreis » verliehen wird). Sørensen hat unseres Wissens in Dänemark als erster einen funk-tionellen Gesichtspunkt auf die Kunst angelegt und sie in einen globalen gesellschaftlichen und ethisch-humanistischen Zusammenhang gestellt. Gleichzeitig hat er eine umfassende literarische Interpretationsmethode geliefert, durch die er die damaligen oft vereinfachenden und unzureichenden Methoden als ungeeignet verwarf. Sørensen nimmt durch seine Ästhetik und literaturkritische Methode eine zentrale Stelle in der theoretischen Diskussion in den 60 er Jahren ein. Die Motivation, die seinen ästhetischen und literaturkritischen Essays zugrunde liegt, ist der Wunsch, andern ein tiefgehendes Verständnis der Literatur im allgemeinen und der modernistischen Literatur im besonderen zu vermitteln. Deshalb übernimmt er 1959 zusammen mit Klaus Rifbjerg die Redaktion der Zeitschrift "Vindrosen », die beide bis 1963 innehaben. Durch diese Tätigkeit trägt er zur Verbreitung sowohl der dänischen als auch der ausländischen modernistischen Literatur in seinem Land bei. Aber auch die persönlichen Kontakte $\mathrm{zu}$ ausländischen Schriftstellern, die er bereits früher aufgenommen hatte ${ }^{2}$ werden in dieser Zeit ausgeweitet. Andererseits hat Sørensen durch die enge Zusammenarbeit mit jungen Kritikern und Studenten, im Rahmen dessen, was Niels Barfoed einmal Sørensens «Ein-Mann-Universität » genannt hat, nachhaltigen Einfluß auf die literarische Seh weise der jüngeren Generation ausgeübt. Z.B. sieht der Kritiker, Essayist und Romanautor Ulrich Horst Petersen in Villy Sørensen seinen direkten Lehrmeister, durch dessen Vermittlung er u.a. großes Interesse für Hermann Broch faßte : Horst Petersen kann als Essayist und Kritiker als 
einer der wichtigsten Erben der Broch-Sørensenschen Literaturauffassung angesehen werden. Wir wollen im Folgenden den künstlerischen, den (Kultur)philosophischen und den ästhetischen Aspekt von Sørensens Werk genauer untersuchen und auf seine initiatorische Bedeutung in diesen drei Bereichen aufmerksam machen.

\section{Sorensens modernistische Erzählungen}

8 Wenn Pil Dahlerup den Durchschlag des Modernismus in Dänemark erst in die 1950-60ger Jahre situiert, dann sind unseres Erachtens folgende Gründe ausschlaggebend gewesen : erstens betrachtet sie die Bewegung, die sich in den 40ger Jahren in der Lyrik gebildet hatte, und deren Organ die Zeitschrift "Heretica " (1948-53) war, nicht als « modernistisch». In der Tat versuchen die Hereticaner durch einen neuen Kult der Lyrik oder gar durch einen neuen Jesus-Kult (Ole Wivel) der Sinnlosigkeit der modernen Welt zu entgehen, während die modernistischen Künstler der nachfolgenden Generation die modernen Bedingungen (Tod sowohl der Transzendenz als der immanenten Wirklichkeit) als einzige Voraussetzung akzeptieren. Zweitens gibt es vor den 1950 er Jahren in Dänemark keine modernistische Prosa. Die naturalistischen Romane, die unter dem Einfluß des «Modernen Durchbruchs» entstanden, behielten die mimetische Ausdrucksweise bei (z.B. Hermann Bang, J. V. Jensen, usw.). Auch in den 1930 er und 1940 er Jahren, in denen im englisch- und deutschsprachigen Raum die ersten großen modernen Romane entstanden (z.B. Joyce, Thomas Mann, Kafka, usw.), war die dänische Prosa noch - von einigen expressionistischen Werken (z.B. Tom Kristensens «Hærværk», 1930) abgesehen - vom realistischen und sozialkritischen Roman beherrscht. Das erste modernistische Prosawerk, das 1953 in Dänemark veröffentlicht wurde, ist der Band «Sære historier » von Villy Sørensen. Übrigens hatten die Redakteure der Zeitschrift Heretica 1952 das Manuskript abgelehnt: sie fühlten sich durch die kompromißlose Darstellung der modernen Problematik in Sørensens Erzählungen abgestoßen.

Worin besteht nun die typische Modernität in Sørensens Erzählungen? Ersten in der Darstellungsweise. Sørensen schreibt phantastische Erzählungen, in denen jeglicher Versuch realistischer Wirklichkeitsabbildung aufgegeben ist: z.B. ist die chronologische Darstellung der Ereignisse dutch eine Art Traumlogik ersetzt, die nach sprunghaften Assoziationen vorgeht: ein Ausdruck ergibt einen anderen, ohne daß eine rational-logische Verbindung zwischen ihnen besteht. Die Sprache selber geht in den Darstellungsprozeß ein, indem sie den Lauf des Geschehens bestimmt, statt ihn bloß abzubilden. Personen oder Orte ermangeln der Detailbeschreibungen, die dem Leser normalerweise ermöglichen, in ihnen individuelle Personen oder bekannte Orte wiederzuerkennen. Sie sind stattdessen nur anhand ihrer für die Aussage entscheidenden Charakteristika gekennzeichnet. Diese Erzähltechnik entspricht dem inneren, psychischen Erlebnis der Gespaltenheit, das in allen Werken zum Ausdruck kommt und das sich bildhaft in zahlreichen Doppelwesen manifestiert: z.B. der siamesische Zwilling Duo in der gleichnamigen Erzählung, die Zwillinge Otto und Otto in der Erzählung "De to tvillinger ", der Gegensatz dunkel und hell als einzige Unterscheidung von zwei gleichnamigen Personen (in «De forsvundne breve»), usw. Man braucht nicht lange zu überlegen, um zu verstehen, daß es sich hier um verschiedene, aber zum Teil verdrängte, Teile ein und derselben Person handelt, vor allem wo wir erfahren, daß diese Personen bis zu einem bestimmten Zeitpunkt unzertrennlich gewesen sind. Ihre schmerzhafte und folgenschwere Trennung läßt sich 
- sehr schematisch - als ein Bild des Menschen deuten, der durch das Leben eines Teils seiner Möglichkeiten beraubt wird, und in dem gerade dadurch der Wunsch erwacht, alle seine Möglichkeiten verwirklichen zu können. Oder anders ausgedrückt : das Leben in der Gesellschaft zwingt den Menschen, einen Teil seiner selbst zu verdrängen, und erweckt dabei gleichzeitig seine Sehnsucht, die dadurch entstandene innere Spaltung zu überwinden. Ob und wie diese Überwindung möglich ist, darüber geben Sørensens Erzählungen keine Auskunft. Sie bleiben bei der schmerzhaften Erfahrung des Konflikts stehen. Thomas Bredsdorff ("Sære fortællere », Kopenhagen, 1967) sieht in der oben dargestellten Problematik den gemeinsamen Nenner einer ganzen Generation von Prosawerken aus dem Jahrzehnt um 1960: der erzähltechnisch weit auseinanderliegenden Novellen und Romanen eines Peter Seeberg, eines Klaus Rifbjerg oder einer Cecil Bødker. Bredsdorff leitet sein Buch mit der Besprechung von Villy Sørensens Werken ein und schließt es durch eine schematische Darstellung der Erzählung «Duo» («Sære historier»), die er als das repräsentativste Werk für die Prosaliteratur dieser Zeit ansieht, ab. Den Titel « Sære fortællere », wählte Bredsdorff unter anderm, weil er in Sørensens Werken « Sære historier» und " Ufarlige historier " die frühesten und vollendetesten Produkte der neuen literarischen Periode fand. Diese Periode endet gegen Ende der 60ger Jahre, wo sie durch zwei gegenläufige Richtungen abgelöst wird: die eine charakterisiert sich durch die Wiederaufnahme des realistischen Erzählstils (die sog. "Neurealisten» wie z.B. Christian Kampmann und Anders Bodeisen), die andere kann als eine Weiterführung des Modernismus angesehen werden (konkretistische oder strukturalistische «Systemdichtung », z.B. Hans-Jørgen Nielsen).

\section{Sørensens kulturphilosophische Überlegungen}

10 « Der Moderne Durchbruch » ist, wir wir eingangs sahen, die Bezeichnung der geistigen Strömung, die in Europa um die Mitte des 19. Jahrhunderts einsetzte, und der der Zusammenbruch einer (politischen, sozialen, religiösen...) Ordnung zugrundeliegt. Sørensen analysiert in seinen Essays diese Krise in der europäischen Kultur aus existentiellpsychologischer Sicht, d.h. er sieht einen engen Zusammenhang zwischen der Entwicklung der einzelnen Persönlichkeit und der Entwicklung der Gesellschaft (mit den wirtschaftlichen, sozialen und kulturellen Aspekten, die dieser Begriff umschließt). Den Zusammenbruch der bestehenden Ordnung (innerhalb derer sich die Gesellschaft im allgemeinen und der einzelne Mensch im besonderen orientiert) nennt Sørensen den Zusammenbruch der Lebensdeutung oder den « Fall ». Indem der Mensch sein Leben deutet, stellt er sich in einen "organischen », gefühlsmäßigen Zusammenhang mit seiner Umwelt. Eine Deutung ist für Sørensen eine « gefühlsmäßige Weltanschauung, mit der man mehr oder weniger zusammengewachsen ist ». Seines Erachtens läßt sich diese Deutung aber nur in der Kindheit aufrechterhalten. In der Pubertät bricht sie zusammen und es entsteht der Konflikt zwischen früher und jetzt. Der Mensch wird sich sozusagen bewußt, daß er nur eine zufällige Erscheinung ist, ein Einzelnes ohne Zusammengehörigkeit mit einer größeren Einheit und ohne eigene Kontinuität. Die moderne Philosophie hat diese Erfahrung die « Konfrontation mit dem Absurden " genannt und das Selbstgefühl des Menschen in dieser Situation als ein «Außer-sich-selbst-Sein » charakterisiert. Sørensen nennt sie die «Spaltung ». Der so gespaltene Mensch versucht natürlich zu einer neuen Einheit mit sich selbst und der Umwelt zu gelangen, die Vergangenheit in die Gegenwart aufzunehmen oder - mit 
Sørensens Wortlaut - sich eine neue Deutung aufzubauen. Was nun das typisch "Moderne » an dieser Situation ausmacht, ist die Tatsache, daß die Gesellschaft ${ }^{3}$ dem $^{2}$ Menschen eine solche "ganzheitliche Deutung » nicht mehr bieten kann oder anders ausgedrückt : die Gesellschaft hat keinen Mythos mehr. Der Mythos ist der Ausdruck des Typischen, des Zeitlosen, des Immerwieder kehrenden. Im Mythos erfuhr der einzelne Mensch, daß sein Leben nichts Zufälliges ist, sondern einer ewigen überindividuellen Gesetzmäßigkeit entspricht. Seine zeitliche und räumliche Begrenztheit wurde im Mythos transzendiert.

11 Wir sagten, daß Deuten ein psychischer (halbbewußt-halbunbewußter) Prozeß ist und eine Deutung (das Resultat dieses Prozesses) eine gefühlsmäßige Weltanschauung : das heißt ein übergeordnetes System von Werten, die den einzelnen Menschen sowohl gefühlsmäßig als intellektuell befriedigen. Ein weiterer Aspekt des « Falls » ist aber die Spaltung zwischen dem Gefühl und dem Intellekt, ersteres ist die instinktive und irrationale Seite des Menschen, letzterer die Seite des rationalen, diskursiven Denkens. Das rationale Denken hat sich Sørensen zufolge in der westlichen Welt schneller entwickelt als z.B. in östlichen Kulturbereichen. Eine Folge dieses hohen intellektuellen Entwicklungsstandes ist die Einsicht, daß das, was wir Transzendenz nennen, keine selbständige metaphysiche Existenz hat, sondern ein Produkt des menschlichen Geistes ist. Folglich haben auch die Werte, die aus dieser Transzendenz abgeleitet wurden, und die deshalb als absolute Werte angesehen wurden, nur noch relative Gültigkeit. Sørensen verweist auf Nietzsche und Marx als die ersten, die den traditionellen Werten ihre metaphysiche Begründung absprechen und sie auf psychologische bzw soziologische Gesetzmäßigkeiten zurückführen. Durch diese Erkenntnis wurde jede mythische Einheitsdeutung des Lebens unmöglich : die letzte war Sørensen zufolge die christlich-mittelalterliche Weltanschauung, die in der humanistisch-bürgerlichen Kultur, in der eine ideale Vorstellung des «Menschlichen» die Rolle Gottes als zentralen Wert übernahm, eine Verlängerung fand. Sørensen betrachtet den « Tod Gottes » als eine Voraussetzung für die Entwicklung der europäischen Gesellschaft zu größerer individueller Freiheit und sozialer Gerechtigkeit; denn erst als der Mensch aufhörte, alle Konflikte im Leben als Vorausbestimmung durch höhere Mächte anzusehen und stattdessen die Ursachen in inneren, psychischen und äußeren, sozialen Bedingungen zu suchen, konnte er aktiv auf seine Umwelt einwirken. Doch die Kehrseite dieser zunehmenden Rationalisierung sieht Sørensen in der Vernachläßigung der irrationalen Seite des Menschen. Denn auch wenn Gott oder das, was der Mensch als das Höchste im Leben ansieht, als eine psychische Projektion entlarvt ist, ist dadurch nicht gesagt, daß diese Projektion nicht einem ursprünglichen Bedürfnis des Menschen entspricht. Dieses irrationale Bedürfnis nach ganzheitlichem Verstehen steht aber im Widerspruch zu dem, was in der modernen Welt die Rolle des Mythos oder der Religion übernommen hat: die wissenschaftliche Erkenntnis. In «Uden mal og med » beschreibt Sørensen dieses Verhältnis folgendermaßen :

Genau wie bei den Mythen, Religionen und Philosophien ist auch bei der Wissenschaft der Drang, das Unbekannte zu bezwingen, indem man es erklärt, die treibende Kraft. Doch ihre Erklärungen sind, so überzeugend sie auch sein mögen, nicht genauso zufriedenstellend. Im Mythos und in der Metaphysik deutet der Mensch, indem er die Natur deutet, zugleich sich selbst; die mehr rationalen Erklärungen, mit denen die Wissenschaft den Menschen versorgt, machendiesen nicht rationaler, sondern machen es ihm schwerer, das Irrationale auszudrücken. Der Unterschied wird klarer, wenn wir die vor-wissenschaftlichen Erklärungen als Deutungenbezeichnen, die wissenschaftlichen als Theorien.Theorien sind Theorien 
über Zusammenhänge zwischen Ursachen und Wirkungen innerhalb begrenzter Bereiche; Deutungen sind Deutungen des Ganzen, und sie sind zielgerichtet: d.h. der Mensch deutet die Dinge mit dem Ziel» ihnen einen Sinn zu geben und er deutet sie, als obsie einen Sinn hätten, einen Zweck erfüllten. Dieser Sinn und der Zweck sind aus den wissenschaftlichen Kausaltheorien ausgeschlossen, und aus diesem Grunde sind letztere weniger zufriedenstellend: sie können zwar das Bedürfnis des Verstands nach Erklärung befriedigen, aber nicht das Bedürfnis des Gefühlslebens nach Sinn, was ursprünglich ein und dasselbe war, aber in der wissenschaftlichen Epoche nicht mehr der Fall ist [...].

(« Uden mål - og med», S. 38).

Die beinahe hundert Jahre, die Sørensen von den Pionieren des «Modernen Durchbruchs" trennen, haben deutliche Spuren hinterlassen. Die naturalistischen Dichter aus dem Kreis Georg Brandes' konnten sich noch vorbehaltlos ihrer Begeisterung für die neuen Möglichkeiten der, vom Joch der Religion befreiten, Wissenschaft hingeben und die Wunder der Technik preisen. Doch spätestens nach dem ersten Weltkrieg erwiesen sich nicht nur die Begrenztheit, sondern auch die Gefahr des rein wissenschaftlichen, positivistischen Denkens. Nicht zuletzt die Tiefenpsychologie hat dazu beigetragen, das europäische Idealbild des rationalen Erkenntnissuchenden zu zerstören und auf bis dahin ungeahnte Kräfte im Menschen aufmerksam zu machen, die - unbeachtet - zur Selbstzerstörung nicht nur des einzelnen Menschen, sondern der ganzen Gesellschaft führen können. (Die zahlreichen massenpsychologischen Schriften, die in den 1930 er-40 er Jahren entstanden, und von denen Hermann Brochs "Massenwahntheorie» als eine der tiefgründigsten anzusehen ist, sind Versuche, dieses letzte Phänomen zu verstehen). Den letzten Stoß erhielt die positivistiche Auffassung der Wirklichkeit von der modernen Physik, die auf die Relativität ihrer eigenen Erkenntnis hinweist. Durch diese neuen Einsichten wird natürlich das Bild einer statischen Wirklichkeit zerstört; die vielgepriesene Immanenz löst sich in unbeschreibbare, und deshalb gefährliche Kräfte auf : « Alle stabilen Größen lösten sich in gefährliche Kräfte auf, die im ersten Weltkrieg Generalprobe hielten » (Villy Sørensen, "Tidens sære kunst», in Aalborg Stiftstidende, 23.5.1960). Diese Kräfte zu bändigen, indem man ihnen Ausdruck verleiht, ist Sørensen zufolge heute genauso dringlich wie in früheren Zeiten.

Um was für Kräfte handelt es sich hier ? Um die unbewußten Kräfte, für die wir keine rationalen Erklärungen haben, aber die nichtsdestoweniger unser Leben und Handeln bestimmen und die wir somit als unserer Wirklichkeit zugehörig betrachten müssen. Das Dilemma des modernen Menschen ist nun die Tatsache, daß er für diese innere Wirklichkeit keinen angemessenen Ausdruck mehr findet. Der rationalisierte und technifizierte Alltag, in dem sich nur noch Spezialisten in ihrem jeweiligen Fachbereich miteinander verständigen können, steht in einem quasi unüberbrückbaren Gegensatz zu dem Bedürfnis nach einer ganzheitlichen Lebensdeutung, in der auch das Irrationale zum Ausdruck kommt.

\section{Wesen und Rolle der Kunst}

Die Rolle der Kunst besteht Sørensen zufolge darin, die irrationalen und unbewußten Teile der Wirklichkeit auszudrücken. Darin ist die Kunst dem Traum ${ }^{4}$ verwandt. Die Bedeutung des Traums für das psychische Gleichgewicht des Menschen ist seit einigen Jahrzehnten wissenschaftlich erwiesen. Und in diesem Beweis sieht Sørensen ebenfalls eine Erklärung für die Funktion der Kunst : "Als amerikanische Wissenschaftler vor 
einigen Jahren entdeckten, daß man wahnsinnig wird, wenn man beim Träumen ständig gestört wird, hatte ich wirklich das Gefühl, daß sich hier eine Erklärung für die Funktion der Kunst befand - die Funktion, die man sonst nie erklären kann » (Villy Sørensen in 33 port cetter, Ninka, Kopenhagen 1969).

Die Funktion sowohl des Traums wie der Kunst besteht darin, auf die unbewußten seelischen Kräfte im Leben hinzuweisen und diese in das bewußte Leben zu integrieren. Der Unterschied zwischen Traum und Kunst ist allerdings die Tatsache, daß die meisten Träume unbewußt und unformuliert bleiben und keine Spuren im wachen Leben hinterlassen, während die Kunst gerade durch ihre Formulierung etwas Neues schafft, das andern Menschen zugänglich ist. In einem Interview sagt Sørensen, daß es nicht auszumachen ist, inwiefern die Künstler « etwas abbilden, was geschieht, oder das, was geschieht, selber schaffen. Vielleicht sind es doch die Künstler, die die allgemeinen Erfahrungen schaffen » (V. Sørensen in Digtere i Forhør, S. 20, Kopenhagen 1966). Das, was geschieht, geschieht im Leben eines jeden Menschen, das Grundmaterial, aus dem die Kunst schöpft, ist also das gleiche wie das, aus dem die Träume entstehen, nämlich das unbewußte Gefühlsleben, das in der Phantasie zum Ausdruck kommt. Sørensen legt großen Wert darauf, die Phantasie, die im Traum und vor allem in der Kunst am Werk ist, von der oft abschätzig benutzten Wendung "das ist reine Phantasie » (womit gemeint ist, das etwas willkürlich und ohne jeglichen Realitätsbezug ist) $\mathrm{zu}$ distanzieren. Die Phantasie ist seines Erachtens nicht willkürlich, sondern unterliegt einer archtypischen Gesetzmäßigkeit, die die Grundformen der Erfahrung bestimmt. Indem der Künstler im Ausdruck seines persönlichen Existenzkonfliktes zu diesen Gesetzmäßigkeiten vordringt, drückt er das Allgemeine im Menschen aus, d.h. die potentiellen Erfahrungsmöglichkeiten, die allen Menschen gemein sind, doch die erst in einer gegebenen Situation verwirklicht werden. In diesem Sinne kann Sørensen schreiben, daß der Künstler die «allgemeinen Erfahrungen schafft» und die Wirklichkeit erweitert. Denn die Symbole des Kunstwerks weisen Neues auf, das wir nicht auf etwas schon Bekanntes zurückführen und begrifflich noch nicht erfassen können.

Wie kann nun Sørensen zufolge der Kunstbetrachter an diesen «allgemeinen Erfahrungen » teilhaben? Nicht anders als daß er, durch das Kunstwerk angeregt, sich für sein eigenes Unbewußtes ${ }^{5}$ öffnet und eine ähnliche Persönlichkeitsentwicklung durchmacht wie die, die er im Kunstwerk vorfindet.

Die eventuelle Wirkung erreicht [der Künstler] gerade dadurch, daß er an die « tieferen Schichten » der Persönlichkeit appelliert, von denen [er] das Gefühl hat, daß er sie in seltenen Augenblicken zumindest teilweise hat ausdrücken können.

(V. Sørensen in Digtere i forhor, S. 32).

Die Funktion der Kunst als Persönlichkeits- und Wirklichkeitserweiterung ist eine allgemeine These der modernistischen Kunsttheorie, die in Dänemark u.a. von dem bedeutendsten Kritiker des Modernismus, Torben Brostrøm, vertreten wird. In «Versets Løvemanke» (1960) schreibt Brostrøm : «In der Kunst holen wir uns selber und die Wirklichkeit, in der wir leben, ein. Man kann heute kein Künstler sein, ohne das "Wissen" seiner Zeit in sich zu haben ". Indem er "Wissen" in Anführungszeichen setzt, deutet Brostrøm an, daß er nicht nur das rationale oder theoretische Wissen meint, sondern auch den irrationalen Gefühlsgehalt. Die moderne Dichtung muß seines Erachtens (und hier stimmt er mit Sørensen überein) über die riesige Spannung zwischen dem bewußten rationalistischen und technifizierten Alltag einerseits und den dunklen Tiefen des unbewußten Seelenlebens andererseits decken (was sie dem 
ungeschulten Leser schwer verständlich macht). Sowohl Brostrøm als Sørensen sehen durch diese Auffassung - im Unterschied zu andern Theoretikern - auch keinen grundlegenden Bruch zwischen dem Naturalismus und dem Modernismus. Sørensen schreibt: "Der Naturalismus wollte Erkenntnis sein, nicht bloß Phantasie [in der Bedeutung "wirklichkeitsfern", der "Modernismus" [...] hat den Erkenntnisbegriff dahingehend erweitert, daß er auch die Phantasie umfaßt » (V. Sørensen in Digtere $i$ forhør, S.22).

Da Sørensen der Kunst eine - wenn nicht erlösende - so doch wegweisende oder erzieherische Rolle in der Gesellschaft beimißt, hat er natürlich den Wunsch, die Kunst auch einem breiten Publikum zugänglich zu machen. Dabei ist es nicht seine Absicht, die Kunst den Wünschen des Publikums anzupassen, sondern dessen Kunstverständnis zu erweitern. Er nimmt deshalb aktiv (durch Vorträge und Zeitungsbeiträge) an der Diskussion um die Berechtigung der Kunst und um eine eventuelle staatliche Unterstützung der Künstler, die sich in den 1960ger Jahren in Dänemarkl abspielt, teil (die sog. "Kunst $f$ onddebatte »). In dieser Diskussion geht es vor allem um die Frage, inwiefern die moderne Kunst noch den Anspruch auf Allgemeingültigkeit erheben kann, wo sie doch angeblich nur wenige Ausgewählte anspricht. Die beiden Vorwürfe, die in der Hauptsache an die modernen Künstler gerichtet werden, sind folgende: erstens die « Unzugänglichkeit » ihrer Werke, die scheinbar mit dem alltäglichen Leben nichts $\mathrm{zu}$ tun haben (Eskapismus), und zweitens deren lebensverneinender und unerbaulicher Charakter (Nihilismus). Stattdessen verlangen die Gegner der modernen Kunst, daß sich die Künstler an die « realistische » Form von Kunst halten sollen.

Es könnte den Anschein haben, daß sich die Auseinandersetzung zwischen den Polen «modernistische » Kunst - «realistische » Kunst abspielt und Sørensen jede Art von realistischer Kunst als schlechte Kunst abtut. In Wirklichkeit kehrt sich Sørensen aber in seiner Verteidigung der modernen Kunst weder gegen die « realistische » Literatur aus der Mitte des 19. Jahrhunderts, noch gegen die neurealistische Literatur seiner eigenen Epoche. Beide sind seines Erachtens gültiger Ausdruck für die sozialen und psychologischen Bedingungen ihrer Zeit, auch wenn sie dadurch, daß sie sich auf die naturgetreue Wiedergabe dieser Bedingungen beschränken, an ihre Zeit gebunden bleiben $^{6}$. Der Gegensatz besteht für Sørensen hingegen zwischen "modernistischer » und «pseudo-realistischer» Literatur. Als pseudorealistische Literatur bezeichnet er erstens die Epigonendichtung (z.B. Unterhaltungsliteratur ${ }^{6}$ ), die die ästhetischen Normen des 19. Jahrhunderts weiterzuführen versucht und dadurch von der modernen Wirklichkeitserfahrung ableitet, und zweitens die Tendenzliteratur, deren Ausgangspunkt eine weltanschauliche Überzeugung ist, die mittels der Kunst propagiert wird.

Sørensens Grundkriterium in der Beurteilung der Kunst ist die Frage nach der ethischen ${ }^{7}$ Haltung, die in dem Werk zum Ausdruck kommt. Von dieser Haltung hängt die ästhetische Qualität des Werkes ab. Sørensen beruft sich in dieser Gleichstellung von Ethik und Ästhetik in der Entstehung eines Kunstwerkes auf Hermann Broch ${ }^{8}$, der ein Kunstwerk ein Kompositum von " ethischer Handlung und ästhetischem Resultat » nennt. Die Grundidee von Brochs Theorie ist folgende : ein guter Künstler wendet sich dem Sinnlosen und Angsterregenden in der Wirklichkeit zu (cf. Ausgangspunkt aller moderner Kunst) und drückt es aus : er formt das Unbekannte. Diesen Formungsakt (der dem Sørenschen Begriff Deuten entspricht) nennt Broch "ethisch», denn er verfolgt kein anderes Ziel, als das offene Suchen nach Wahrheit. Das völlig neue 
Produkt, das aus diesem Prozeß hervorgeht, wird als schön empfunden, und schön ist ein anderes Wort für ästhetisch. Gute Kunst ist also Broch zufolge ethisch und ästhetisch zugleich. Schlechte Kunst (z.B. Epigonendichtung) dagegen, ist nicht etwa schlecht, weil sie unästhetisch ist, sondern im Gegenteil weil sie nur ästhetisch ist und dadurch unethisch. Der schlechte Künstler formt die Wirklichkeit nicht so, wie sie sich ihm darbietet, sondern so, wie er oder der Leser sie sich wünschen. Dabei greift er auf etwas bereits Geformtes zurück, das er reproduziert (z.B. versucht er anhand von stilistischen Kniffen und sprachlichen Klischees eine bestimmte Stimmung hervorzurufen). Das, was bei einem guten Kunstwerk als spontanes Resultat hervorgeht, wird in der schlechten Kunst zu einem erklärten Ziel.

21 Es handelt sich bei dieser Art von Kunst immer um sentimentale Kunst, die Sørensen zufolge die Wirklichkeit umdeutet, statt zu einer neuen Deutung vorzudringen. Eine sentimentale Haltung (ob im Kunstschaffen oder im Leben im allgemeinen) besteht darin, die Gefühle, die in einer Konfliktsituation entstehen (Zusammenbruch der alten Lebensdeutung), und die ob ihrer Unbekanntheit meistens angenehm und unangenehm zugleich sind, nicht wahrhaben zu wollen und sich stattdessen nach den alten noch konflikt freien Gefühlen zurückzusehnen. Was dadurch ausgedrückt wird, sind keine wirklichen Gefühle - denn ein Gefühl ist immer auf etwas außerhalb des eigenen Ichs bezogen - sondern das, was Sørensen « Fühlerei » nennt : man fühlt sich selber fühlen. Solange ein Kunstwerk von diesen rein "privaten" Gefühlen des Künstlers nicht befreit ist, d.h. solange die neuen Gefühle nicht in seine Persönlichkeit intergriert sind und nur noch diese veränderte Persönlichkeit zum Ausdruck kommt, kann das Werk nicht als gelungen angesehen werden und geht sowohl Broch als Sørensen zufolge keinen andern Menschen etwas an.

22 Neben der sentimentalen Dichtung verweigert Sørensen aber auch der Tendenzliteratur die Bezeichnung Kunst. In dieser Art von Dichtung wird zwar der ursprüngliche Konflikt nicht einfach verdrängt, aber er wird auch nicht offen und ehrlich zum Ausdruck gebracht, sondern er wird lediglich dazu benutzt, eine vorgefaßte theoretische Überzeugung des Autors zu dokumentieren. Sørensen nennt diese Art von Literatur "Umwertung ». Zur Erläuterung müssen wir noch einmal die Bedeutung der Erkenntnisfunktionen «Deuten» «und Bewerten» in Erinnerung rufen : « Deuten » ist ein fortlaufender psychischer Prozeß, in dem der Mensch ständig neue Synthesen zwischen seinem irrationalen Gefühlsleben und rationalen Denken schafft und der sich nur in Form von Symbolen Ausschlag gibt. Kunst ist der Ausdruck eines solchen Deutungsprozesses. "Bewerten" ist die darauffolgende begriffliche Formulierung der eventuell erlangten Erkenntnis ${ }^{9}$ und somit eine bewußte Stellungnahme: wissenschaftliche, philosophische und ideologische Theorien sind «Bewertungen". "Bewertungen» sind aber nur die statischen Resultate eines Erkenntnisprozesses, die, wenn sie nicht ständig erneuert werden, zu Konventionen erstarren. Die Kunst, die nun ihren Ausgangspunkt in einer solchen theoretischen Bewertung nimmt, versperrt sich selber den Weg zu neuer Wirklichkeitserkenntnis und folglich $\mathrm{zu}$ neuen Bewertung en derselben. Ihre vermeintliche Bewertung der Wirklichkeit ist lediglich eine Umwertung derselben. Solche Kunst ist immer tendenziös. Die bekanntesten Beispiele von Tendenzliteratur sind Sørensen zufolge die realsozialistische Literatur der (ehemaligen) Ostblockländer und die katholische Literatur (als dänische Exponente dieser letzten Richtung sieht er die Romane Jakob 
Knudsens an, in denen seines Erachtens der tendenziöse Aspekt mit privatpsychologischen Umdeutungen einhergeht).

Sørensen wendet sich mit seinem ethisch-ästhetischen Gesichtspunkt auf die Kunst gegen zwei geläufige Beurteilungsmethoden, die beide das Wesen der Kunst verkennen: die "rein ästhetische» und die "moralische ». Der rein ästhetische Gesichtspunkt besteht darin, ein Kunstwerk lediglich auf die Einlösung gewisser formaler Anforderungen hin zu untersuchen (z.B. formalistische Kritik). Dadurch wird die Auffassung verstärkt, daß Kunst zwar unterhaltsame, aber harmlose - weil wirklichkeitsferne - Spielerei ist. In ihrem extremsten Aspekt besteht diese Haltung darin, die Kunst als eine Sache des Geschmacks zu betrachten, wodurch jegliches Beurteilungskriterium verschwindet.

zweite Kunstbetrachtung, gegen die sich Sørensen in der oben dargestellten Theorie wendet, ist die « moralische ». Diese Kritik fragt im Gegensatz zur ästhetischen ausschließlich nach den inhaltlichen « Meinungsäußerungen » (im Sinne moralischer, religiöser oder politisch-ideologischer Überzeugungen), die im Werk zum Ausdruck kommen. Auch eine solche Kritik verfälscht das Wesen der Kunst und verstößt gegen deren Déontologie, die Sørensen zufolge darin besteht, die Verfolgung kunstexterner Ziele zu untersagen. Der Künstler kann als Privatperson noch so überzeugende Meinungen vertreten, in seinem Werk sind diese Meinungen fehl am Platz. Hier kommt es nur auf die aus dem dargestellten Konflikt entstehende « Haltung » an.

Der Einheit von ethischem und ästhetischem Aspekt beim Kunstschaffen wird in der Kritik Rechnung getragen, indem Inhalt und Form zusammen als ein organisches Ganzes betrachtet werden.

Die Komposition ist kein sekundäres Arrangement eines fertig-konzipierten Werks, sie gehört zum Schaffungsprozeß selber.

(V. Sørensen, « Digtere og dæmoner », S. 39)

Sørensen plädiert also für eine werkimmanente Deutung, die die Autonomie des Kunstwerks respektiert: dazu muß sich der Kritiker erstens von seinen eigenen « privaten » Gefühlen befreien, d.h. er muß sich seiner selbst soweit bewußt sein, daß er nicht (wie viele naive Leser) unbewußt seine ungedeuteten Gefühle in das Werk projiziert. Oder anders gesagt: «der Kritiker muß vernehmen können, was bei ihm selber echt ist, um beurteilen zu können, was in einem Kunstwerk echt ist » (mündliche Aussage Sørensens in einem Interview mit der Verfasserin des vorliegenden Artikels, März 1991). Die Intuition des Kritikers spielt also für Sørensen eine große Rolle. Darüberhinaus muß sich der Kritiker ebenfalls von seinen persönlichen Anschauungen befreien und sich von der Sympathie bzw. Antipathie, die er der Person des Autors entgegenbringt, lossagen.

Wie läßt sich nun die Unechtheit eines Kunstwerkes aufdek-ken? An Brüchen in der Form, d.h. ästhetische Schönheitsfehler verweisen Sørensen zufolge immer auf Mängel in der grundlegenden «Haltung » (in dem persönlichen Deutungsprozeß, für den das Kunstwerk Ausdruck ist).

In diesen beiden grundlegenden Aspekten seiner Literaturkritik steht Sørensen dem « New Criticism » nahe : der literaturkritischen Methode, die in den angelsächsichen Ländern gleichzeitig mit der modernistischen Literatur entstand, und hauptsächlich auf diese angewandt wurde. Sørensen war einer der ersten, der in Dänemark nach " neukritischen » Prinzipien verfuhr ${ }^{10}$, ohne allerdings in allem mit dem New Criticism übereinzustimmen. Seine wichtigste Abweichung vom New Criticism besteht darin, daß 
er sich nicht auf reine Textanalyse beschränkt, sondern ein literarisches Werk auch als Ausdruck für soziologische und psycho-soziologische Bedingungen seiner Zeit ansieht, vor allem da er sich als Kritiker hauptsächlich mit Romanen beschäftigt.

\section{NOTES}

1. Zu dieser Zeit waren Brochs Werke im deutschsprachigen Raum noch fast unbekannt.

2. 1957 war er z.B. bei dem (west)deutschen Schriftsteller- und Kritikerverbai. « Gruppe 47 » zu Gast.

3. Die politische und soziale Entwicklung der europäischen Gesellschaft ab dem 19.Jahrhundert hat Sørensen in zahlreichen Essays behandelt. Wir können diesbezüglich u.a. auf seine Einleitung zu Karl Marx, « Økonomi og filosofi » (1962), sowie auf « Hverken-eller» (1961) und « Meilern fortid og fremtid» (1969) verweisen.

4. In dieser Auffassung des Traums steht Sørensen der analytischen Psychologie C.-G.Jungs nahe, derzufolge die Traumsymbole nicht nur auf vergangene unbewußte Ereignisse im Leben des Träumenden zurückweisen, sondern eine ordnende und zielgerichtete Funktion haben, durch die dem Leben Richtung und Sinn gegeben wird.

5. Sørensen weist darauf hin, daß von den sog. "realistischen" Romanen aus dem 19. Jahrhundert für den modernen Leser nur noch diejenigen eine Bedeutung haben, die nicht rein realistisch sind (z.B. Dostojewski), d.h. deren Symbolik sich nicht auf die Konventionen ihrer Zeit reduzieren läßt, weil sie in tiefere Schichten weist.

6. Die modernistischen Künstler und Kritiker beziehen die Produkte der Massenmedien (Radiosendungen, Filme, Reklame, usw.) in ihren Tätigkeitsbereich ein. Z.B. führt Klaus Rifbjerg in der Zeit als er, zusammen mit Villy Sørensen, die Zeitschrift Vindrosen redigiert, dort eine feste Filmrubrik ein, in der u.a. der Name Ulrich Horst Petersen sehr oft anzutreffen ist.

7. Der Begriff "ethisch» ist streng von dem Begriff «moralisch» zu unterscheiden. Eine " ethische" Haltung ist Offensein für das Unbekannte und Unformuherte (das eine sowohl anziehende als abschreckende Wirkung auf den Menschen hat) sowie der Wille, dieses Unbekannte zu integrieren. Eine « moralische » Haltung dagegen besteht darin, das Unbekannte unter Berufung auf konventionelle (Gesellschafts) Normen zu unterdrücken.

8. Siehe Hermann Broch, «Schriften zur Literatur 2 », 1975. Hier vor allem das Kapitel « Kitsch und Literatur ".

9. Erkenntnis bedeutet bei Sørensen nichts anderes als « in eine echtere Beziehung zur inneren und äußeren Wirklichkeit zu gelangen». Die Tiefenpsychologie nennt diesen Prozeß Individuation.

10. Für ausführlichere Informationen über die dänische Neukritik verweisen wir auf Johan Fjord Jensen, « Den ny kritik », Kopenhagen, 1962. 


\section{RÉSUMÉS}

Der Dichterphilosoph Villy Sørensen hat sich in mehreren Bereichen des dänischen Modernismus der 1950ger-60ger Jahre als Galionsfigur erwiesen.

Mit seinem ersten Band Erzählungen «Sære historier» (1953) befreit er die Prosa aus den Zwängen der realistischen und naturalistischen Ästhetik und leitet eine fruchtbare Periode modernistischer Novellen- und Romandichtung ein.

1959 liefert er mit «Digtere og daemoner » eine der ersten theoretischen Gesamtdarstellungen der modernen Epoche ab dem ausgehenden 19. Jahrhundert und ihrer philosophischen und literarischen Erzeugnisse. Dadurch trägt er einerseits zur Einführung oder zumindest zur Neuinterpretierung von europäischem Gedankengut aus den vergangenen Jahrzehnten, aber auch aus seiner eigenen Zeit, in Dänemark bei. Andererseits schaffter durch seine persönliche Synthese von ausländischen Einflüssen und der Beobachtung seiner eigenen kulturellen, politischen und literarischen Umgebung eine neue Grundlage für die intellektuelle Aktivität seiner dänischen Zeitgenossen.

Auf dem Gebiet der Kunst gibt sich dies in der Aufstellung einer allgemeinen Literaturtheorie sowie einer neuen literaturkritischen Methode Ausschlag, die der modernistischen Literatur kritik der 60ger Jahre zugute kommen. Der Grundgedanke Sørensens ist (wie der Hermann Brochs), daß die Kunst ästhetisch und «ethisch» zugleich sein muß, d.h. das Resultat einer «ethischen» Handlung, die ohne Vorurteile nach der Wahrheit strebt, indem sie dem «Unbekannten » in der Wirklichkeit symbolischen Ausdruck verleiht.

Dans les années 50-60, le philosophe-écrivain Villy Sørensen apparait comme la figure de proue de la prose moderniste danoise à plusieurs égards.

Par son premier recueil "Sære historier», il libère la prose des contraintes de l'esthétique réaliste et inaugure une série de nouvelles et de romans modernistes dans son pays.

L'essai «Digtere og dæmoner» (1959) constitue une synthèse théorique des œuvres philosophiques et littéraires européennes depuis la fin du XIX siècle et des propres observations de Sørensen sur son environnement politique, culturel et littéraire. Cette synthèse jette les bases d'un renouveau intellectuel pour les jeunes danois de l'époque.Au plan esthétique, il en résulte une nouvelle théorie de la littérature et une nouvelle méthode critique dont s'inspirera la critique littéraire moderniste des années soixante au Danemark. L'idée fondamentale est (comme chez Hermann Broch) que l'art doit être à la fois esthétique et « éthique ", c'est-à-dire le résultat " esthétique » d'une action « éthique », celle qui recherche sans préjugés la vérité, en exprimant sous forme symbolique "l'inconnu» de la réalité. Cette conception contribuera de manière significative au débat des années soixante sur le rôle de l'art dans la société moderne.

\section{AUTEUR}

\section{HEDWIG REUTER}

Université de Mons - Belgique 